\title{
Design of Wireless Embedded Vibration Data Acquisition Terminal
}

\author{
Zhiyuan Sun, Xinsheng Ma \\ Institute of Engineering Mechanics of China Earthquake Administration, Harbin, 150080, China \\ sunzhiyuaniem@126.com
}

Keywords: Wireless, Vibration data, smart sensor network.

Abstract. Structural health monitoring of large civil engineering has been a hot point of research in the world these years. As one of the key parts, design and implement of structural vibration data acquisition system in actual projects is more and more valued for people. Monitoring technology as the core of newly-developing wireless communication has also been widely extended and used in structural health monitoring. This essay aimed at widely using of wireless technology in smart sensor network and introduced the design of embedded terminal which serves for smart collection system based on embedded technology and modern rapid development network platform.

\section{Introduction}

With wireless technology being mature, more and more industriesbegin using wireless network to carry on real-time long-distance monitoring. Modern structural health monitoring combines wireless sensor network technology, mobile communication technology, Wi-Fi technology, embedded system technology, and smart information processing technology, which is applied to monitor structural performance, detect variation of structure, and reveal damage of structure and degradation of structural performance[1].It has always been a basic method in structural engineering to measure structural strain, acceleration, speed, displacement, torsion and so on. Because of the particularly of civil engineering, such as various of structure form, long working cycle, complex influence factor of structure performance and so on, its monitoring is very complex.Most of the health monitoring and damage recognition technology is based on structural vibration information and finds out injury in the early-stage by technology such as pattern recognition, which could confirm the injury position within sensor resolution, judge damage level, and predict structural surplus valid lifetime[2]. The vibration data collection and processing system that is often installed in structure to be monitored consists of computer and network and collects vibration parameter translated by sensor and deals them with smoothing and amplification. The communication system will transmit the collected and proceeded data to monitoring center[3].

These years, embedded system in industrial manufacture has been widely used. On the level of device in field instrument and monitoring using embedded technique is development tendency of monitoring network. Embedded technique does not only supply developer with a lot of tools and function library which will reduce amount of traditional clients and workload of doing secondary development of network clients, but also combines embedded technique and internet technique which makes the whole control network have universal base communication protocol and is good for network's seamless link compared with field bus technology. With this technology we can monitor field data and devices at any time and any place in the monitoring center[4]. In this essay, we used AM3359 as the client controller chip with embedded Linux real-time operation system as manage system for the device. 


\section{Hardware Design}

The wireless embedded vibration collection system terminal consists of primary embedded device, data collection board and Wi-Fi wireless data transmission module.

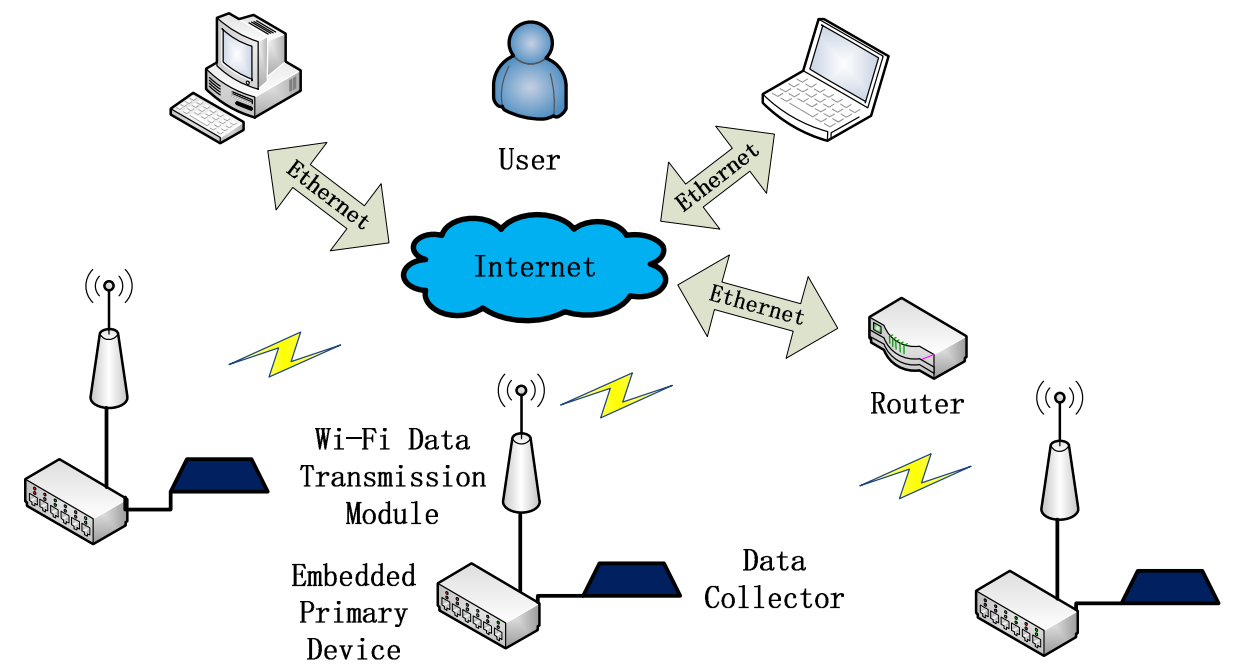

Fig.1. Structure of Wireless Embedded data collection system

The primary embedded device connects Wi-Fi wireless data transmission module with internet ports and the data collection board communicates with primary embedded device via SPI serial port. Eventually, data gets access in Internet network via wireless router.

\section{Hardware design of primary embedded device}

The hardware platform of embedded device mainly relies on Texas Instrument Cortex-8 based AM3395 microprocessor, which is better use for picture and imagine processing, peripheral application and industrial interface such as Ether CAT and PROFIBUS. The AM3395 works at high speed clock frequency and supports general-purpose memory (NAND, NOR, SRAM, etc.). It supports up to 16-bits ECC and dual-port 10/100/1000 Ethernet switch. The AM3395 is considered to be a specific product positioning MCU for industry controlling and has plenty of accessible public information. It supports several operating systems such as Linux, Android, etc. and has extensive use in every aspect. Its peripheral hardware circuit includes driving circuit of internet ports, power supply circuit, and storage circuit. The structure of hardware circuit is shown in Fig. 2. 


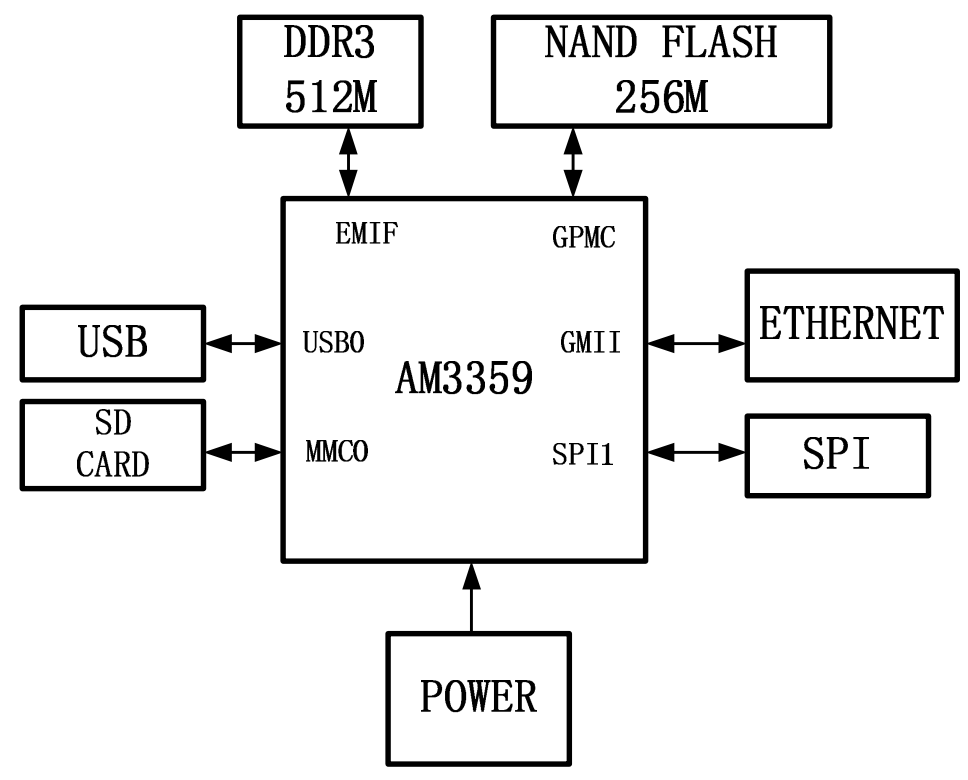

Fig.2.Structure of primary hardware circuit

There is MAC module built in the AM3395, but we need to access a PHY chip to realize Ethernet data transmission. Underlying driving of Ethernet is to operate PHY chip. In this design we use DP83848 chip to achieve control of Ethernet PHY[5]. Meanwhile, we choose MT41K256M16HA-125chip as DDR3 RAM of embedded system. The storage space is small due to the size of chip, so signal input need to be amplified to ensure its efficiency. This amplification is accomplished by S-AMP and one memory stack corresponds to one S-AMP channel.

Nonlinear microcell mode inside Nand Flash, it provides a valid and cheap solution for solid high-capacity memory. Nand Flash memory has advantage of high capacity and high speed of rewriting. Because of that, Nand Flash has been more and more widely used[6]. We use such memory medium to store booting system and lead system starting from Nand Flash. We use Samsung K9F2G08X0B chip as its hardware.

To prevent generating error code and losing code leading to incomplete information during period of data transmission, we need to storage much vibration information in the field. So we just add external storage card (SD CARD) function in this design in order to conveniently backup and transplant data.

\section{Hardware design of vibration data acquisition}

The core chip of vibration data acquisition is ADI AD7606, which is a 16-bits multi-channel synchronous analog-to-digital converter. In the meantime, vibration data acquisition uses Colibrys VS9002 chip to collect object's acceleration in three dimensions. Its measuring range is $\pm 2 \mathrm{~g}$ and its sensitivity could reach a level of $1000 \mathrm{mV} / \mathrm{g}$. Its measuring bandwidth is $250 \mathrm{~Hz}$, so it has high resolution in low frequency and is fit for vibration monitoring aspect in civil engineering.However, the signal tested in environment is pretty poor, it has to be smoothed and amplified before analog- 
to-digital conversion to make sure data have good precision. In this design we use integrated opamp to build up filter and amplifier whose cut-off frequency is $100 \mathrm{~Hz}$. Fig. 3 shows structure of data acquisition circuit.

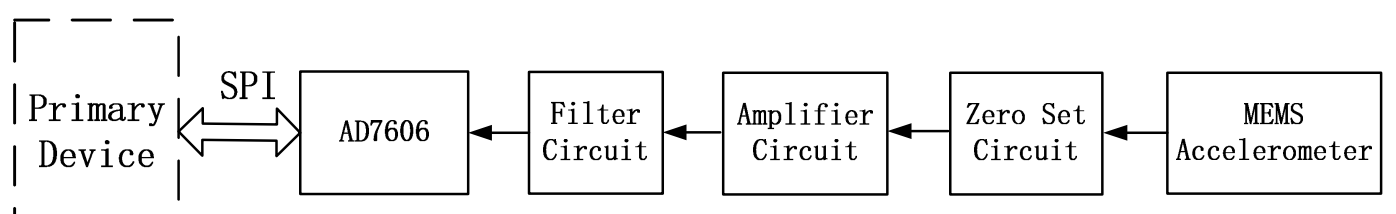

Fig.3.Structure of data acquisition circuit

The chosen AD7606 contains an analog input clamp protection, a track-and-hold amplifier, a second-order antialiasing analog filter, a 16-bit change redistribution successive approximation analog-to-digital converter(ADC), a digital filter, a $2.5 \mathrm{~V}$ voltage reference, a voltage reference buffer and high speed parallel and serial interfaces. In the meanwhile, all channels can sample at throughput rate of $200 \mathrm{kSPS}$. The AD7606 has $1 \mathrm{M} \Omega$ analog input impedance regardless of the sampling frequency. The antialiasing analog filter has a $3 \mathrm{~dB}$ cut-off frequency of $22 \mathrm{kHz}$; When sampling at $200 \mathrm{kSPS}$, it will provide $40 \mathrm{~dB}$ antialias rejection. The ADC channels of AD7606 are controlled by two CONVST signal, which CONVST A is used to control channel V1 V4. ADC will initiate simultaneous sampling while there is a rising edge of CONVST. The sampling hold circuitry is set to hold indicated that the conversion process has started. Then the BUSY output remains high and when the falling edge of BUSY comes, the conversion process gets completed, which also means that data is available to read from the digital output. AM3359 is designed to read data through SPI series interfaces in this work.

\section{Software design}

By writing multithreading underlying drive program in Linux, integrated management and processing of Ethernet data can be realized. During data processing and saving threading, the program records received local and Ethernet data in the form of files in seconds[7]. These files and data are used to re-draw historic waves. Through multithreading, the software can not only ensure the implement of system sampling frequency, data processing and save, but also ensure instantaneity.

In Linux, network programming is implemented by Socket(). When designing programs by TCP, due to the fact that TCP protocol is a reliable, facing connection, end-to-end based on IP protocol, server side and client side need to be designed. Client side plays an important role in this work---get access to upper computer. During the data access, interface function will experience a series of process, such as monitoring connection, TCP recv function and send function, connecting confirming function, connecting close function, SOCKET close function. The work mainly introduces software 
design of this process; specific TCP/IP communication flow diagram is shown in figure 4.

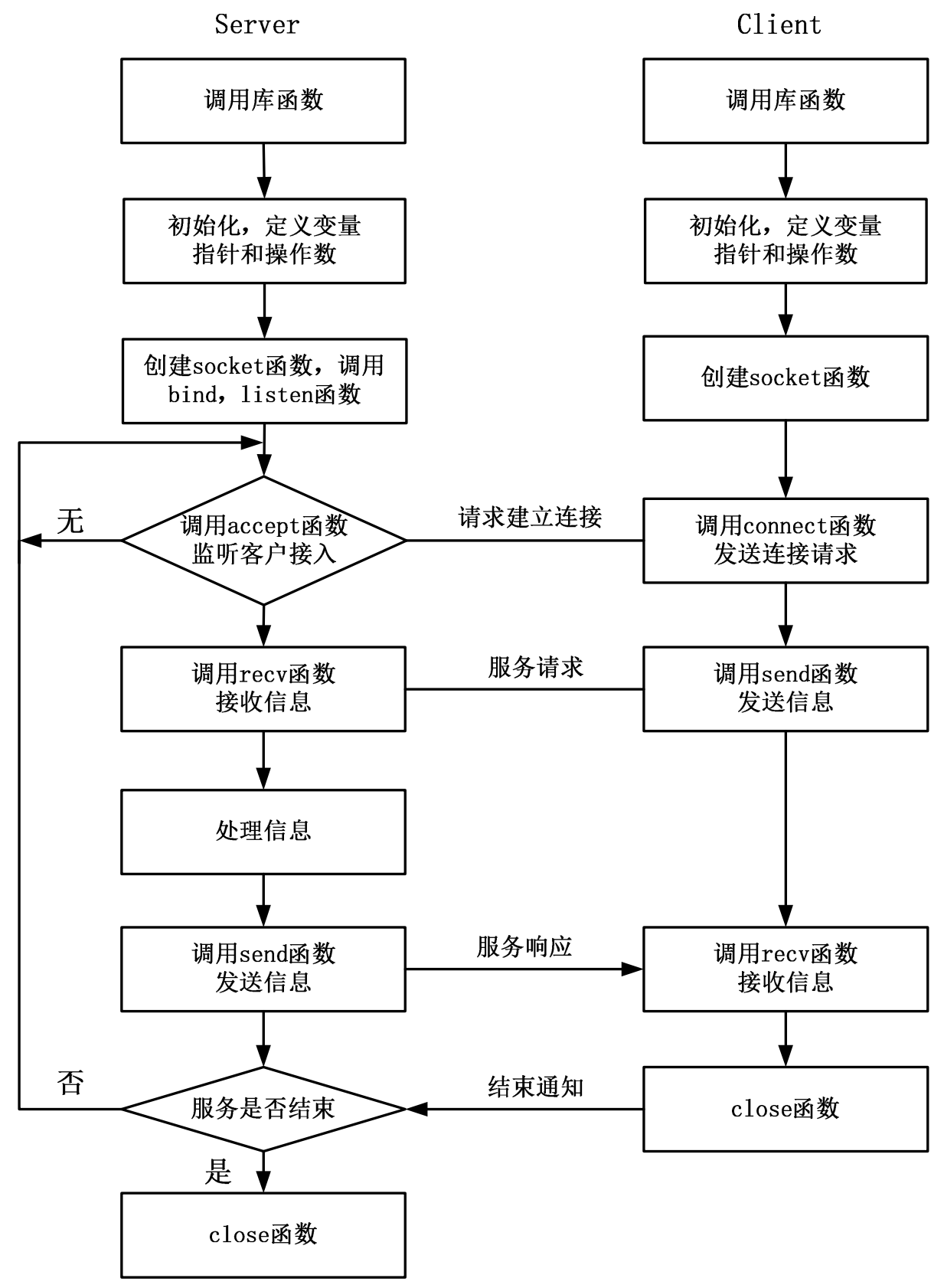

Fig.4.TCP/IP communication flow diagram

\section{Conclusion}

Embedded collection terminal designed for vibration measurement has the characteristics of simple environment, easy to maintain and lower cost. Embedded terminal design concept and model can play an efficient role in various physical quantities collection and transmission. Transmission media can be changed according to environment and security requirements. Transportprotocols can also develop user-defined-design embedded based on requirement. Just like the paper use embedded micro-processor of TCP protocol connect upper computer through Ethernet. In practical engineering projects, embedded wireless vibration data acquisition based on AM3359 in this work can take 
advantages of low-layer embedded micro-processor in data collection, saving and communication management, so that it can satisfy the basic using requirement of general structure multi-node health monitoring instantaneously, which has an extensive application prospect.

\section{Acknowledgements}

This work was financially supported by the Fundamental Research Funds for the Central Public Institutes of Institute of Engineering Mechanics of China Earthquake Administration (No.2014B05).

\section{References}

[1] Aktan A.E: Issues in infrastructure health monitoring for management. Journal of Engineering Mechanics Vol. 126 (2000), p. 711-724.

[2] Ko J M, Ni Y Q.: Technology developments in structural health monitoring of large-scale bridges . Engineering Structures Vol.56(2005), p. 1715-1725.

[3] LI Hong-nan. Safety assessment: Health monitoring and damage diagnosis for structures in civil engineering . Earthquake Engineering and Engineering Vibration Vol.22(2002), p. 82-90.

[4] Ying Lu: Development Technology and Application of Embedded System(Tsinghua University Press, China 2005).

[5] Ligong Zhou: ARM Embedded System Foundation Coursebook (Beihang University Press, China 2005).

[6] Zhengrong Liu, Zhichao Zhang, ZhenshanXu: Embedded Linux Application Development Explanation (China Machine Press, China 2004).

[7] Mark G.Sobell in:: A Practical Guide to Linux Commands, edtied by Zhan Bao, Xianghuakong, Gensheng Hu, Publising, Beijing, China (2010), in press. 